\title{
Imaging of turbulent structures and tomographic reconstruction of TORPEX plasma emissivity
}

\author{
D. Iraji, I. Furno, A. Fasoli, and C. Theiler \\ Centre de Recherches en Physique des Plasmas (CRPP), Ecole Polytechnique Fédérale de Lausanne \\ (EPFL), Association EURATOM-Confédération Suisse, CH-1015 Lausanne, Switzerland
}

(Received 22 July 2010; accepted 11 November 2010; published online 8 December 2010; publisher error corrected 13 December 2010)

In the TORPEX [A. Fasoli et al., Phys. Plasmas 13, 055902 (2006)], a simple magnetized plasma device, low frequency electrostatic fluctuations associated with interchange waves, are routinely measured by means of extensive sets of Langmuir probes. To complement the electrostatic probe measurements of plasma turbulence and study of plasma structures smaller than the spatial resolution of probes array, a nonperturbative direct imaging system has been developed on TORPEX, including a fast framing Photron-APX-RS camera and an image intensifier unit. From the line-integrated camera images, we compute the poloidal emissivity profile of the plasma by applying a tomographic reconstruction technique using a pixel method and solving an overdetermined set of equations by singular value decomposition. This allows comparing statistical, spectral, and spatial properties of visible light radiation with electrostatic fluctuations. The shape and position of the time-averaged reconstructed plasma emissivity are observed to be similar to those of the ion saturation current profile. In the core plasma, excluding the electron cyclotron and upper hybrid resonant layers, the mean value of the plasma emissivity is observed to vary with $\left(T_{e}\right)^{\alpha}\left(n_{e}\right)^{\beta}$, in which $\alpha=0.25-0.7$ and $\beta=0.8-1.4$, in agreement with collisional radiative model. The tomographic reconstruction is applied to the fast camera movie acquired with $50 \mathrm{kframes} / \mathrm{s}$ rate and $2 \mu \mathrm{s}$ of exposure time to obtain the temporal evolutions of the emissivity fluctuations. Conditional average sampling is also applied to visualize and measure sizes of structures associated with the interchange mode. The $\omega$-time and the two-dimensional $k$-space Fourier analysis of the reconstructed emissivity fluctuations show the same interchange mode that is detected in the $\omega$ and $k$ spectra of the ion saturation current fluctuations measured by probes. Small scale turbulent plasma structures can be detected and tracked in the reconstructed emissivity movies with the spatial resolution down to $2 \mathrm{~cm}$, well beyond the spatial resolution of the probe array. () 2010 American Institute of Physics. [doi:10.1063/1.3523052]

\section{INTRODUCTION}

Plasma particle and heat losses associated with turbulence are among the most addressed physics issues in fusion research, as they may have considerable effects on particle and energy confinement time in the future fusion reactors, including ITER. ${ }^{1}$ Understanding the nature of turbulence is an important research line in magnetic fusion physics, ${ }^{2}$ and one that can benefit significantly from experiments on basic plasma physics devices. ${ }^{3}$ In fusion devices, measurements of edge turbulent structures, such as blobs and filaments, mainly come from single-point, multipoint probe or optical measurements, ${ }^{4,5}$ although recently two-dimensional measurements were obtained using internal Langmuir probes array. ${ }^{6}$ The strongest limitation of internal probes is that they can only be used when they do not significantly perturb the plasma and when they are not destroyed by the plasma. Ideally, one would need to perform a full spatiotemporal imaging of the turbulence with adequate spatial and temporal resolutions and without perturbing the plasma. Fast framing cameras satisfy these conditions as they can monitor light emission without any perturbation, and are now commonly used to study turbulence in plasmas. ${ }^{7-9}$

To study the fundamental aspects of the physics of plasma turbulence, a Toroidal Plasma Experiment (TORPEX) was developed at the CRPP. ${ }^{10}$ The TORPEX device allows the production of well-diagnosed reproducible plasmas, which feature properties similar to tokamak edge plasmas, including turbulent structures like blobs ${ }^{11}$ and statistical properties of fluctuations. ${ }^{12}$ Low frequency electrostatic fluctuations, associated with ideal and resistive interchange modes, are routinely measured by means of extensive sets of Langmuir probes. A hexagonal array of 86 electrostatic probes, dubbed HEXTIP (HEXagonal Turbulence Imaging Probes), provides a full coverage of the plasma crosssection and allows imaging of turbulent structures of the ion saturation current in the spatiotemporal domain. ${ }^{13}$ SLP (Slow Langmuir Probes), a linear array of 8 Langmuir probes, can measure time-averaged profiles of the electron density and temperature by shot to shot scanning of the poloidal cross-section. ${ }^{14}$

An imaging system, including a Photron Ultima APX-RS fast framing camera and a Hamamatsu C10880-03 image intensifier, has been developed to complement electrostatic measurements. With the addition of the visible fast camera, it is possible to compare the statistical and spectral properties of probe measurements in one plasma cross- 
section, with the passive completely nonperturbative and high spatiotemporal resolution optical reconstruction.

In this work, the tomographic reconstruction of the plasma emissivity profile in the TORPEX open magnetic field configuration is performed. The plasma visible emission is imaged by the fast camera in a tangential view. To reconstruct the poloidal emissivity profile, a pixel method is considered and an optimized reconstruction procedure is applied. This technique enables us to visualize and analyze small scale turbulent plasma structures in TORPEX. The tomographic reconstruction of TORPEX plasma emissivity also provides insight into the plasma light emission mechanisms when combined with HEXTIP and other probes. The two-dimensional (2D) fluctuation profiles can be measured entirely nonperturbatively for frequencies up to $20 \mathrm{kHz}$, and a spatial resolution down to $2 \mathrm{~cm}$, well beyond HEXTIP spatial resolution. We show that the properties of light fluctuations are the same as those measured with HEXTIP.

This paper is organized as follows. The theory of the reconstruction technique is discussed in Sec. II. The tomographic reconstruction of fast camera images on TORPEX and the experimental setup are explained in Secs. III and IV, respectively. Section V discusses an optimized reconstruction of the time-averaged profile of the plasma emissivity profile as well as a cross validation with HEXTIP and SLP data, and the dependence of the plasma emissivity upon the electron density and temperature. Section VI addresses the reconstruction of fluctuation profiles of the plasma emissivity and the cross validation with HEXTIP data using conditional average sampling technique and Fourier analysis. Conclusions are given in Sec. VII.

\section{THEORETICAL BACKGROUND OF PLASMA TOMOGRAPHY}

Provided that the plasma is fully transparent to its radiation, the plasma light emission is integrated along lines of sight (LOSs) passing through different plasma regions. The signal of each pixel of the image results from a combination of light coming from different plasma regions. In general, a line-integrated image (LII) of the plasma emissivity may result in errors in the size, shape, position, and speed of the plasma structures. A tomographic reconstruction of the plasma emissivity profile from the LII can, in principle, overcome this problem. In plasma physics, particularly in fusion related researches, several attempts have been performed to use the tomographic reconstruction to gain information about plasma rotation, mode structures, MHD (MagnetoHydroDynamics) activities, and current profile in tokamaks. ${ }^{15-18}$

Tomographic reconstruction is usually performed with measurements obtained from different views. This may be difficult and expensive, particularly in the case of fast imaging. As an alternative approach, the tangential view of the plasma column by means of only a single fast camera can provide enough information for the tomographic reconstruction under the assumption of plasma homogeneity along magnetic field lines. This approach is adopted in this work.

Common digital cameras, which are used for plasma imaging, provide images that are inherently discretized into

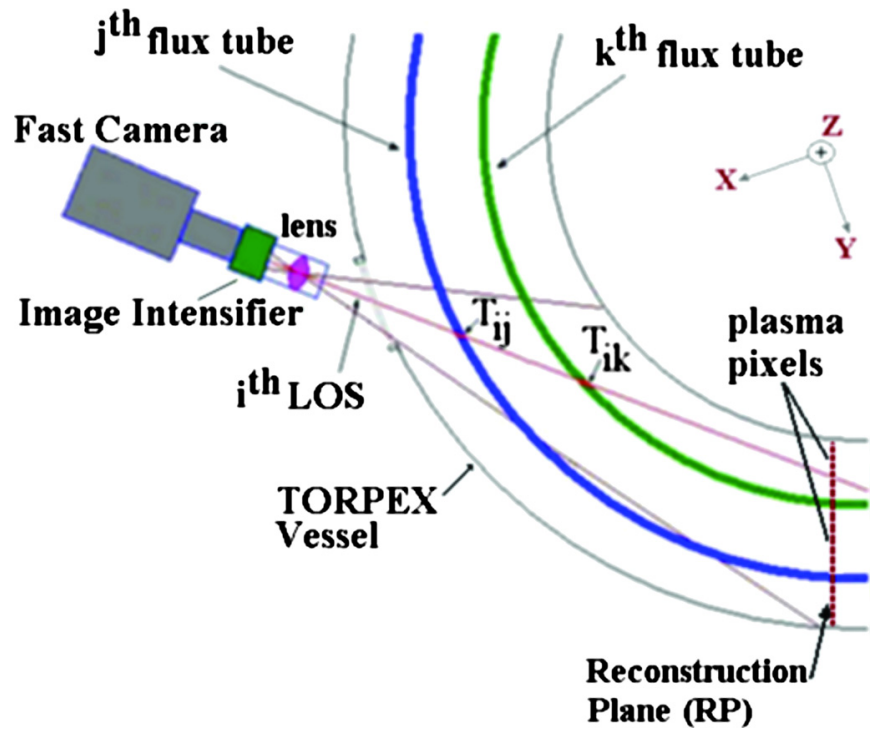

FIG. 1. (Color online) Schematic of the tangential imaging of plasma in TORPEX and the tomographic reconstruction. $T_{i j}$ and $T_{i k}$ are lengths of intersection of $i$ th LOS with $j$ th and $k$ th flux tubes corresponding to the $j$ th and $k$ th plasma pixels. RP is a poloidal plane of the torus on which the objective lens of the camera is focused is defined as the reconstruction plane and $C_{\mathrm{RP}}$ is the center of the RP.

identical pixels. To perform the tomographic reconstruction, the poloidal cross-section of the plasma is also divided into cells, which will be referred to as plasma pixels. A poloidal plane of the torus, on which the objective lens of the camera is focused, is defined as the reconstruction plane (RP), schematically shown in Fig. 1. Each plasma pixel is a footprint of a flux tube on the RP. Each flux tube follows magnetic field lines and the plasma pixel term also refers to the corresponding flux tube. Plasma pixels may have different shapes. In previous works, circular plasma pixels were considered. ${ }^{19}$

In this work, the poloidal cross-section of the vacuum vessel is divided into square plasma pixels with area of $p$ $\times p \mathrm{~mm}^{2}$. The lowest limit of $p$ depends upon the robustness of the tomographic reconstruction technique against noise and quality of the images. The size of the plasma pixels $(p)$ should be small enough to satisfy the assumption of homogeneity of plasma radiation inside one particular plasma pixel and should also be smaller than any characteristic scale lengths $(L)$ in the plasma, $p \ll L$. On the other hand, $p$ should be larger than the width of the LOS. The total power $\left(P_{i}\right)$ received by detector number $i$ can be computed using the well known formula ${ }^{19}$ as follows:

$$
P_{i}=\varepsilon_{i} \sum_{j=1: N} T_{i j} g_{j} .
$$

Here $\varepsilon_{i}$ is the etendue of the $i$ th detector, $g_{j}$ is the emissivity $\left(\mathrm{W} / \mathrm{m}^{3} / \mathrm{sr}\right)$ of the $j$ th plasma pixel, $N$ is the number of plasma pixels on the poloidal cross-section, and $T_{i j}$ is the corresponding length of intersection of the $i$ th LOS with $j$ th plasma pixel (see Fig. 1). Therefore, the brightness (power/ etendue) of the $i$ th detector is given by 


$$
I_{i}=\sum_{j=1: N} T_{i j} g_{j}, \quad i=1: M .
$$

$M$ is the number of the camera image pixels. In compact form, the set of simultaneous linear equations in Eq. (2) is written in the form of matrix multiplication,

$$
\mathbf{I}=\mathbf{T} * \mathbf{g} .
$$

where $\mathbf{T}, \mathbf{g}$, and $\mathbf{I}$ are, respectively, the transfer matrix, the emissivity, and the brightness profiles. Finding a proper solution for $\mathbf{g}$ is the main goal of tomographic problems. The set of linear equations represented by Eq. (2) expresses an underdetermined $(M<N)$ or overdetermined $(M \geq N)$ set of linear equations. The residual square $\left(R^{2}\right)$ is defined as $R^{2}$ $=(\mathbf{T} * \mathbf{g}-\mathbf{I})^{\prime} *(\mathbf{T} * \mathbf{g}-\mathbf{I})$, where $\left({ }^{\prime}\right)$ indicates matrix transposition. $\mathbf{g}$ has to be found in such a way that $R^{2}$ goes to zero. For underdetermined systems, $R^{2}=0$ and there is an infinite number of solutions for $\mathbf{g}$. To find the best fit $\mathbf{g}$, different methods such as linear regularization, Phillips-Tikhonov regularization, Cormack inversion, maximum entropy, and minimum Fisher information methods were developed. ${ }^{15,20-22}$ In the case of an overdetermined system, a stable solution can be found by minimizing the $R^{2}$ value using least squares fitting methods. One of the most useful methods to do least squares fitting is singular value decomposition (SVD), ${ }^{23}$ which is used in this work.

\section{TOMOGRAPHY ON TORPEX}

The TORPEX device has a major radius $R=1 \mathrm{~m}$, a minor radius $a=0.2 \mathrm{~m}$, a mainly toroidal magnetic field $\left(B_{t}\right)$ up to $0.1 \mathrm{~T}$, and a small vertical magnetic field component $B_{z}$ $\leq 4 \mathrm{mT}$, which is superimposed to partly short circuit the vertical electric field due to $\nabla \mathbf{B}$ and curvature drifts and reduce the particle losses. This magnetic configuration results in open field lines with a pitch angle that depends upon the ratio $B_{z} / B_{t}$. Highly reproducible plasmas are created and sustained for up to $3 \mathrm{~s}$ by means of electromagnetic waves in the electron cyclotron range of frequency of $2.45 \mathrm{GHz}$ and a power up to $20 \mathrm{~kW}$ produced by a magnetron microwave source. ${ }^{10}$ In this work, a fast visible camera (Photron Ultima APX-RS) is used to image TORPEX plasmas. ${ }^{24}$ The camera is capable of recording an image every $4 \mu \mathrm{s}$ at maximum frame rate and benefits from a $1024 \times 1024$ array of $17 \mu \mathrm{m} \times 17 \mu \mathrm{m}$ CMOS (Complementary-symmetry MetalOxide-Semiconductor) image sensors. The camera is aligned tangentially with respect to the toroidal direction, as schematically shown in Fig. 1, and is located in the equatorial plane of the torus looking through a lateral window between two toroidal magnetic field coils. The camera is equipped with a Nikon (NIKKOR, $50 \mathrm{~mm}$, f/1.4) objective lens, which is used at $f / 2$ aperture and can cover almost $70 \%$ of the plasma cross-section. The imaging system is tested for the vignetting effect using a uniform light source. In this work, the vignetting effect is negligible since the plasma is imaged on a small area of the camera chip. Therefore, only the central part of the lens and consequently of the intensifier's photocathode are used. In the case of framing rates faster than 10 kframes/s, the camera is equipped with an image intensifier unit (Hamamatsu, model: C10880-03) (Ref. 25) and a 1:1 relay lens. No interference filter is used and the camera chip detects visible light in the range of 400-700 nm with averaged quantum efficiency of $40 \%$.

Each camera pixel covers an area of $0.06 \times 0.06 \mathrm{~cm}^{2}$ on the reconstruction plane. In practice to provide a large depth of field, the camera image is averaged over several pixels. Therefore, each image pixel covers a larger area than a single camera pixel. The maximum size of the image pixels as mapped on the reconstruction plane in the vicinity of the optical axis of the objective lens is $0.6 \times 0.6 \mathrm{~cm}^{2}$. The maximum size of the mapped image pixels on the vessel wall is $0.9 \times 0.9 \mathrm{~cm}^{2}$. Therefore, the size of plasma pixels $(p)$ should be larger than $0.9 \mathrm{~cm}$ and has to be small to guarantee the validity of the plasma homogeneity inside a plasma pixel and having adequate spatial resolution of the reconstructed emissivity profile. Also the size of plasma pixels $(p)$ determines the number of plasma pixels and the number of unknowns in the reconstruction problem $(N)$. In addition, the number of detectors is equal to the number of equations $(M)$. This gives additional constrain to the plasma pixel size $p$ in such a way that $M>N$, leading to an overdetermined problem. Finally, the tomographic reconstruction is performed with the plasma pixel size of $p=1 \mathrm{~cm}$.

To calculate $T_{i j}$, the equation of corresponding LOS is solved assuming that the LOS of the ith detector passes through the center of the objective lens. In practice, the equation for the $i$ th LOS is written considering the position of the $i$ th detector on the camera chip and the center of the objective lens. A Cartesian coordinate system is used in such a way that its origin is the center of the machine, the XY plane fits on the equatorial plane of the torus, and the $\mathrm{X}$ axis intercepts the center of the imaging window (Fig. 1). Therefore, the position of the camera detectors is computed considering the alignment and the position of the camera. The position of the center of the RP $\left(C_{\mathrm{RP}}\right)$ is obtained considering the position of the center of the objective lens and its focal distance. As a result, the positions of all plasma pixels on the RP are determined and the trajectory of each flux tube that passes through the corresponding plasma pixel is calculated according to the pitch angle of the magnetic field line. To calculate the trajectory of each flux tube, the vertical and toroidal coil currents (respectively $I_{\mathrm{ver}}$ and $I_{\mathrm{tor}}$ ) are measured with errors less than $1 \%$ and the magnetic field components for every point across the vessel are calculated using sophisticated routines, which were developed for TORPEX. The vacuum magnetic field configuration as computed from the TORPEX routines has been cross-checked with experimental measurements, performed with a Hall probe. To compute the transfer matrix (T), only those LOSs, which go through the window, are considered. Two intersection points of the LOS, the one with the window and the other with the vacuum vessel wall, are calculated. The full LOS length, starting from the first intersection point with the window and ending on the inner vessel wall, is divided into small segments of length $d l$. Knowing the exact position of the center of $l_{k}$ (the $k$ th segment on the LOS) determines the flux tube in which $l_{k}$ lies. The number of the segments inside the intersection of the $i$ th LOS with the corresponding flux tube of the $j$ th plasma pixels is counted as $\left(u_{i j}\right)$. Finally, $T_{i j}$ is obtained by 
simulated HEXTIP profile ( $0 \%$ noise) simulated HEXTIP profile ( $5 \%$ noise)
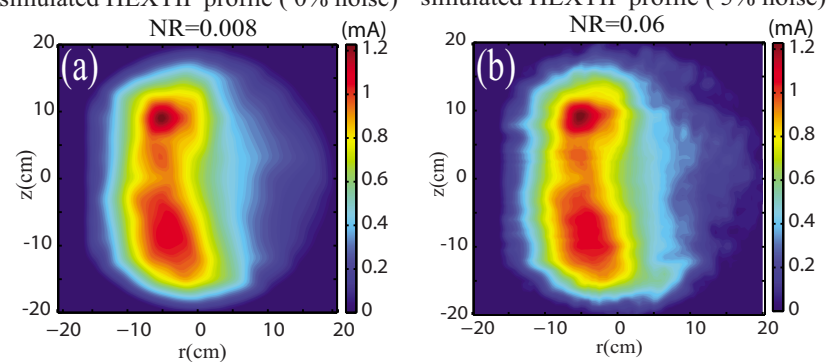

simulated HEXTIP profile ( $10 \%$ noise) simulated HEXTIP profile ( $20 \%$ noise)
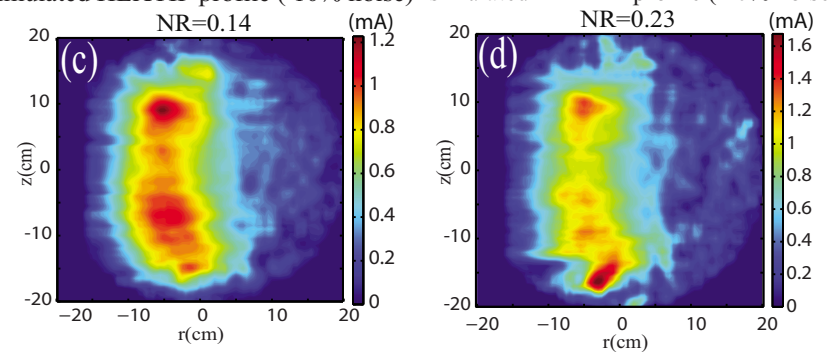

FIG. 2. (Color online) Four simulated HEXTIP profiles obtained by adding different levels of noise to the line-integrated original HEXTIP profile. (a)(d), respectively, are reconstructed by adding $0 \%, 5 \%, 10 \%$, and $20 \%$ of the local value as amplitude of random noise to the line-integrated profile.

$$
T_{i j}=u_{i j} d l .
$$

Once $T$ is calculated, the SVD method is used to calculate $\mathbf{g}$. To quantify the quality of reconstruction, a normalized residual (NR) is defined as

$$
\mathrm{NR}=\sqrt{\sum_{i=1: M}\left\{\left[\sum_{j=1: N}\left(T_{i j} g_{j}\right)-I_{i}\right] / I_{i}\right\}^{2}} .
$$

Here $g_{j}$ is the value of the $j$ th pixel in the reconstructed emissivity profile and $I_{i}$ corresponds to the $i$ th pixel of the camera image. The NR is obtained by summing over all the camera image pixels and plasma pixels. In this work, the typical relative error of electrostatic probe measurements of 0.2 (Ref. 26) is considered as the upper limit of the NR to test the quality of the tomographic reconstruction. The transfer matrix (T) is calculated in the case of $p=1 \mathrm{~cm}$ and applied to the time-averaged plasma emission profile (I) to get the time-averaged reconstructed emissivity profile (g).

To estimate the noise effect upon the reconstructed profiles, two kinds of noises are considered: the electronic noise, which is produced by the camera and the image intensifier, and the wall reflection. To reduce the first to a negligible level, before each shot the background electronic noise is subtracted automatically by the camera. The remaining electronic noise is scintillation noise, which is produced by the multichannel plate of the image intensifier. The level of the random scintillation noise (RSN) depends upon the incident light intensity and gain voltage which is about $5 \%$ in this work. To test the robustness of the reconstruction method against random noise level, the HEXTIP profile $(\mathbf{H})$ is considered as a reference. The line-integrated profile $\left(\mathbf{H}_{L}\right)$ is computed as $\mathbf{H}_{L}=\mathbf{T} * \mathbf{H}$. The reconstructed profile $\left(\mathbf{H}_{R}\right)$ of HEXTIP is calculated using the reconstruction procedure. Figure 2 shows four simulated-reconstructed HEXTIP pro- files, which underline the robustness of the tomographic reconstruction against different levels of noise. In this case, different levels of random noise are added to the lineintegrated HEXTIP profile $\left(\mathbf{H}_{L}\right)$. Then the $\left(\mathbf{H}_{R}\right)$ is retrieved back for each noise level. A similar approach is used to quantify the reflection noise effect upon the reconstructed profile. To approximate the reflection noise pattern, the projection of the line-integrated profile $\left(\mathbf{H}_{L}\right)$ on the wall is multiplied with a reflection coefficient $(\mathrm{RC})$. The reflection noise pattern is added to the line-integrated profile and finally the tomographic reconstruction is performed for different values of the $\mathrm{RC}$. As a result, the analysis shows that for values of RSN and $\mathrm{RC}$, respectively, smaller than $10 \%$ and $30 \%$, the NR does not exceed 0.2 , thus resulting in a well reconstructed emissivity profile. Similarly, to estimate the total noise effect $(\mathrm{RSN}+\mathrm{RC})$ upon the spatial resolution in the reconstructed profiles, an additional test is performed considering synthetic 2D Gaussian profiles with the FWHM synthetic $_{\text {varying from } 1}$ to $20 \mathrm{~cm}$. These synthetic structures are considered to correspond to the plasma emissivity profiles. Different levels of the total noise are considered and the FWHM reconstructed $_{\text {of }}$ reconstructed profiles are obtained and compared with those of synthetic 2D Gaussian profiles $\left(\mathrm{FWHM}_{\text {synthetic }}\right)$. This analysis shows that up to $\mathrm{RSN}=5 \%$ and $\mathrm{RC}=20 \%$ the relative error $\left(E_{\mathrm{FWHM}}=\mathrm{FWHM}_{\text {synthetic }}\right.$ $\left.-\mathrm{FWHM}_{\text {reconstructed }} / \mathrm{FWHM}_{\text {synthetic }}\right)$ is less than $10 \%$ for structures in which $\mathrm{FWHM}_{\text {synthetic }}<12 \mathrm{~cm}$. Therefore, the spatial resolution in the reconstructed emissivity profile is only limited by the plasma pixel size $(1 \mathrm{~cm})$ to $2 \mathrm{~cm}$ due to Nyquist theorem.

\section{EXPERIMENTAL RESULTS}

In this work, an interchange dominated hydrogen plasma scenario is considered. In these plasmas, the time-averaged density and temperature profiles are quasislab-like (i.e., vertically elongated) and the plasma density fluctuations are mostly associated with an interchange plasma mode. This configuration has been extensively studied by means of HEXTIP, SLP, and other probe-based diagnostics. ${ }^{27}$ These plasmas are produced by $400 \mathrm{~W}$ of magnetron power and the average magnetic field consists of a vertical magnetic field (2.3 $\mathrm{mT})$ superimposed to the dominant toroidal component (77 mT). Hydrogen is injected into the vacuum chamber with a pressure up to $3.5 \times 10^{-5}$ mbar. Typical values of the electron density and temperature are $n_{e} \sim 10^{16} \mathrm{~m}^{-3}$ and $T_{e}$ $\sim 5 \mathrm{eV}$, respectively. To image the plasma, the camera is equipped with the image intensifier and acquires at 50 $\mathrm{kframes} / \mathrm{s}$ for $200 \mathrm{~ms}$. The intensifier is gated with the camera shutter signal for $2 \mu s$ with the repetition rate equal to the framing rate of the camera. The gain voltage of the intensifier is set to $800 \mathrm{~V}$ resulting in average photon gain of $3 \times 10^{3}$ (photons/photon).

\section{RECONSTRUCTION OF TIME-AVERAGED PROFILES}

Figure 3(a) shows the time-averaged plasma light emission, tangentially imaged by the camera. The reconstructed local emissivity profile is shown in Fig. 3(b). The normalized 

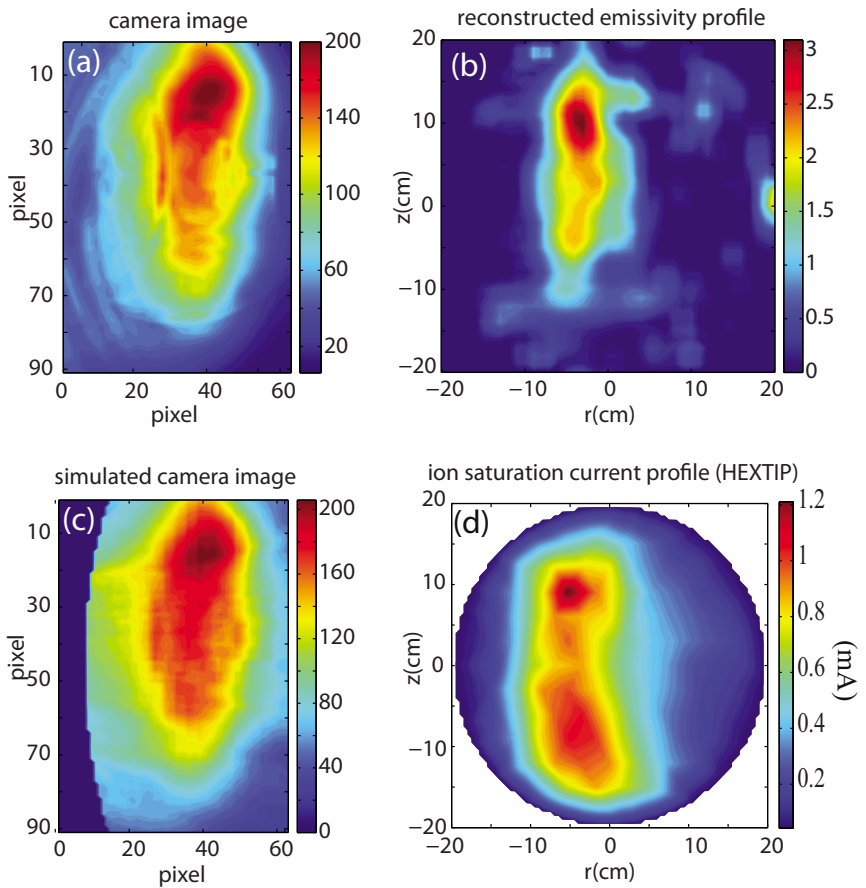

FIG. 3. (Color online) (a) Tangentially viewed time-averaged image of the plasma column. (b) Plasma emissivity profile reconstructed from the tangentially viewed image. (c) Simulated camera image obtained by line integrating the reconstructed plasma emissivity profile in (b). (d) Time-averaged ion saturation current profile measured by HEXTIP.

residual of this reconstruction is about 0.1 . Figure 3(c) shows the simulated tangentially viewed plasma image, considering the reconstructed profile, Fig. 3(b) as the plasma emission profile. The simulated camera image shows quite good agreement with the original camera image. Figure 3(d) shows the time-averaged ion saturation current $\left(I_{\text {sat }}\right)$ profile measured by HEXTIP. The plasma emission profile and the ion saturation current profile are in good agreement in terms of shape and position. The radial profiles of the normalized time-averaged emissivity and the ion saturation current are shown in Fig. 4. The radial profiles are obtained by $z$-averaging over the full height of the HEXTIP and emissivity profiles.

Both profiles peak at $z=10 \mathrm{~cm}, r=-4 \mathrm{~cm}$ but the radial full width at half maximum (FWHM) of the emissivity profile is smaller than that of the density profile by a factor of 0.5. This figure shows similarities between HEXTIP profile and the reconstructed emissivity, particularly in the mode region. The ion saturation current is proportional to the electron density and square root of the electron temperature. Therefore, Fig. 4 indicates that in the mode region, the plasma emissivity dependence upon the electron density and temperature is similar to that of the ion saturation current.

To investigate the dependence of the emissivity upon the electron density and the electron temperature, these parameters are measured directly by moving the SLP shot by shot over the poloidal cross-section. ${ }^{14}$ Figures $5(\mathrm{a})$ and $5(\mathrm{~b})$ show the time-averaged electron density and temperature profiles. These electron density and temperature profiles are repeated several times for different experiments.

According to the collisional radiative $(\mathrm{CR})$ model, ${ }^{28}$ the

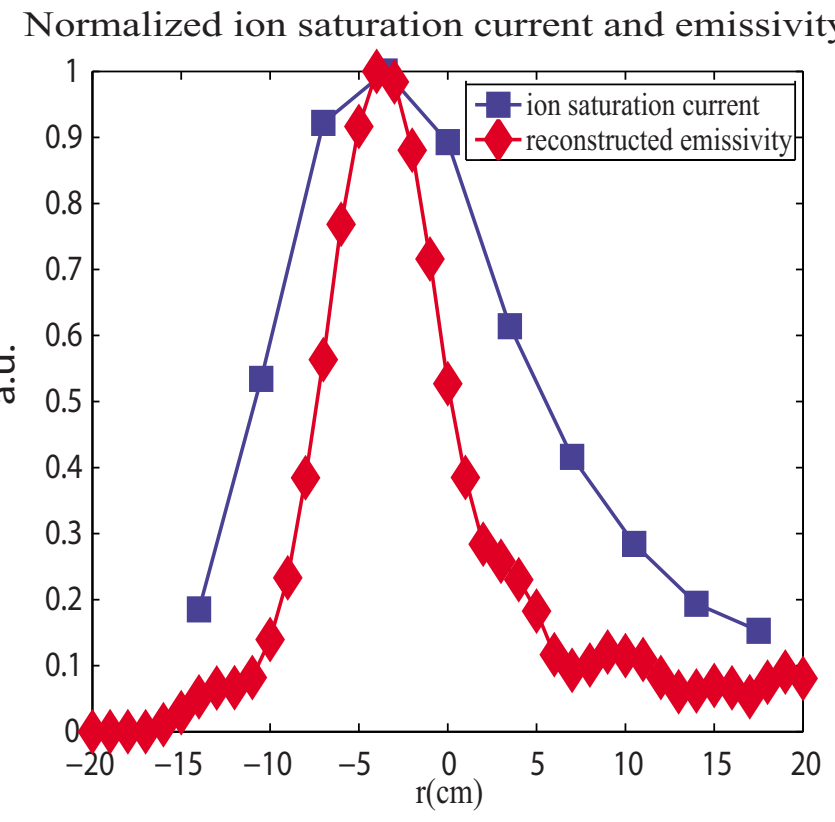

FIG. 4. (Color online) Normalized $z$-averaged ion saturation current and reconstructed emissivity profiles.

plasma emissivity dependence upon the electron density and temperature could be modeled as $g \propto\left(T_{e}\right)^{\alpha}\left(n_{e}\right)^{\beta, 5,8}$ The 2D electron density and temperature profiles are considered to compute $\alpha$ and $\beta$ by fitting the function $\gamma\left(T_{e}\right)^{\alpha}\left(n_{e}\right)^{\beta}$ to the reconstructed emissivity profile $(\mathbf{g})$. Here $\left(T_{e}\right),\left(n_{e}\right)$, and $(g)$ are, respectively, the $2 \mathrm{D}$ profiles of the electron temperature, the electron density, and the reconstructed emissivity. Assuming a flat neutral density profile, $\gamma$ is supposed to be a constant parameter independent of the electron density and temperature. Figures 6(a) and 6(b) show the obtained 2D profiles of $\alpha$ and $\beta$. The values of $\alpha$ and $\beta$ are, respectively, about $0.25-0.7$ and $0.8-1.4$ in the region $-8 \mathrm{~cm} \leq r$ $\leq-3 \mathrm{~cm}$ and $-5 \mathrm{~cm} \leq z \leq 5 \mathrm{~cm}$. The $\gamma$ profile is almost constant in this region. In Fig. 6(c) the simulated radial profile of the emissivity using $\alpha_{\mathrm{av}}=0.35$ and $\beta_{\mathrm{av}}=0.9$ as well as

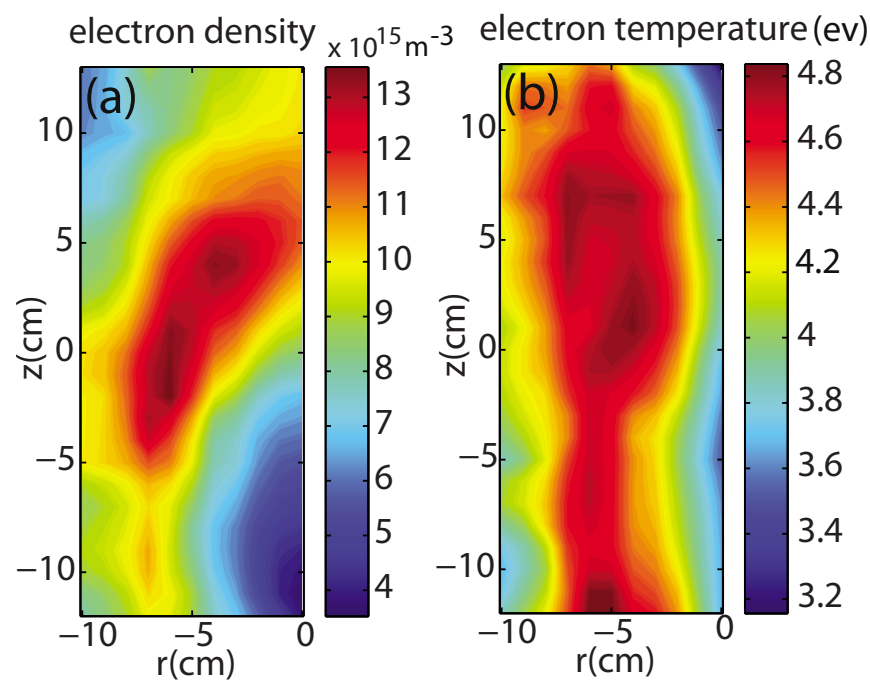

FIG. 5. (Color online) (a) Time-averaged electron density profile measured by SLP. (b) Time-averaged electron temperature profile measured by SLP. 

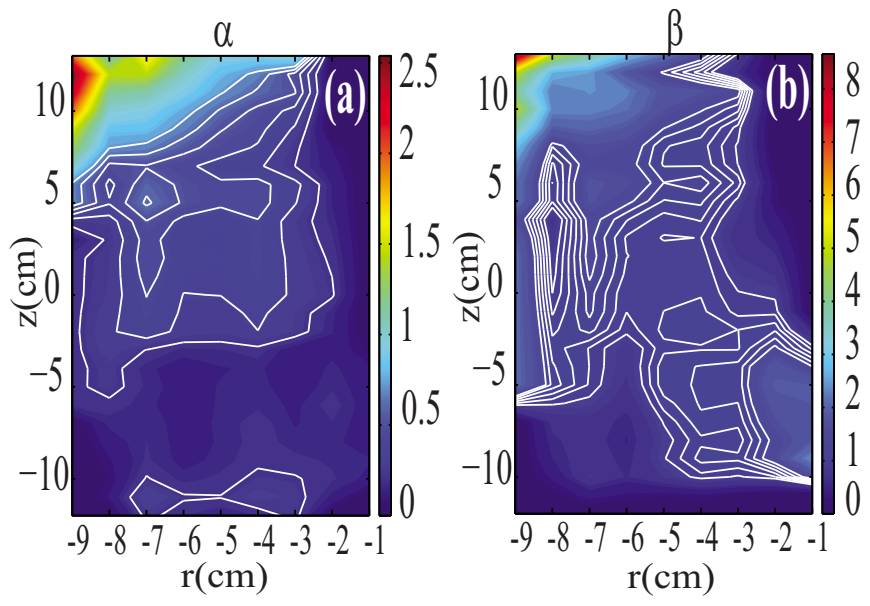

measured and simulated radial profile of emissivity

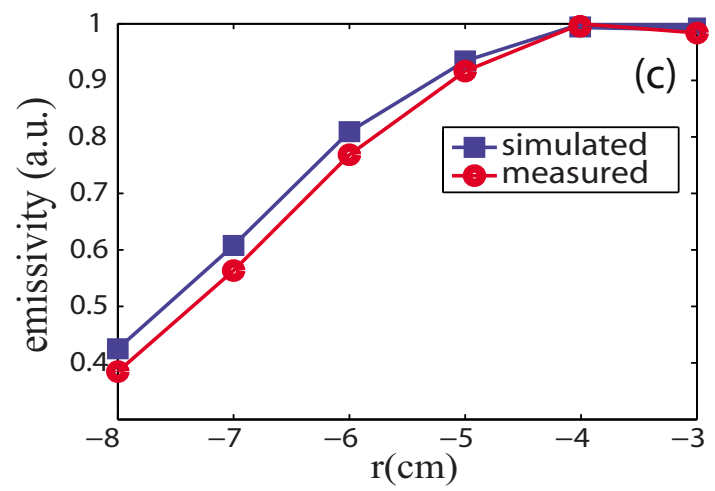

FIG. 6. (Color online) [(a) and (b)] 2D profile of $\alpha, \beta$ obtained by fitting of 2D profiles of the electron density and temperature $\left(T_{e}\right)_{r}$ and $\left(n_{e}\right)_{r}$ to the reconstructed emissivity profile $(g)_{r}$ assuming homogeneous neutral density. (c) Simulated radial profile of the emissivity using $\alpha_{\mathrm{av}}=0.35$ and $\beta_{\mathrm{av}}=0.9$ and measured radial profile of the emissivity.

the radial profile of the measured emissivity is shown for $-8 \mathrm{~cm} \leq r \leq-3 \mathrm{~cm}$. This is the region where the $\alpha$ and $\beta$ profiles are smooth and their values are compatible with the CR approximation.

\section{RECONSTRUCTION OF FLUCTUATIONS PROFILE}

The camera and other diagnostics in TORPEX are synchronized, and after a plasma discharge, data are retrieved from the camera memory and used to compare with probe signals and to perform the analysis. To obtain the temporal evolution of the fluctuations profile, the tomographic reconstruction is first performed frame by frame. Then the profile of the fluctuations is computed by subtracting the timeaveraged reconstructed profile. Depending on the spatial resolution of the reconstruction, the size of images, and the number of frames, this procedure can be time consuming. In this work, tomographic reconstructions of $200 \mathrm{~ms}$ of the camera movie (10 000 frames) are performed. Reconstruction of each frame takes approximately $14 \mathrm{~s}$ using a $2.6 \mathrm{GHz}$ processor. In Figs. 7(a) and 7(b), four consecutive frames of the camera movie as well as the corresponding four reconstructed plasma emissivity profiles are shown. The exposure time is $2 \mu$ s with $50 \mathrm{kframe} / \mathrm{s}$ repetition rate. These are adequate to image and to track the turbulent plasma structures (a). Camera images (before tomographic reconstruction)
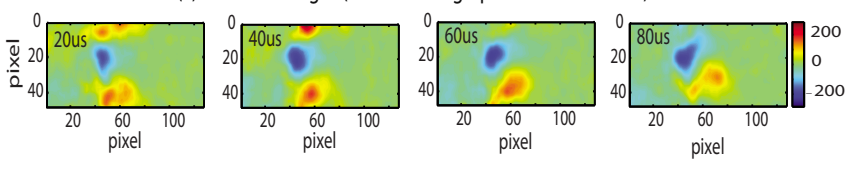

(b). After tomographic reconstruction
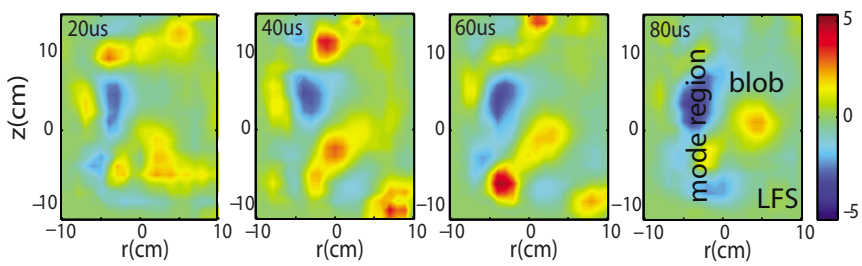

FIG. 7. (Color online) (a) Four consecutive frames of camera images before tomographic reconstruction. (b) Four corresponding reconstructed plasma emissivity profiles showing the detachment of a blob. The LFS stands for the low field side.

in TORPEX plasmas. These frames show the evolution of a plasma blob. In the camera movie frames before the tomographic reconstruction, due to the line integration, the blob seems to stay attached to the mode region, as shown in the fourth frame of Fig. 7(a). However, the tomographic reconstruction reveals that the blob is a detached structure that moves radially outward. This is clearly visible in the third and fourth frames of Fig. 7(b). This is consistent with the previous results from Langmuir probe data in similar plasmas. ${ }^{11,27}$

The radial profile of power spectrum density (PSD) of the ion saturation current fluctuations is calculated by taking the signals of all HEXTIP probes at the vertical position $z=0 \mathrm{~cm}$. Similarly, the radial profile of the PSD of the reconstructed emissivity fluctuations is obtained by taking the signals of the pixels at $z=0 \mathrm{~cm}$ between $r=-6 \mathrm{~cm}$ and $r=+7 \mathrm{~cm}$.

Results are shown in Figs. 8(a) and 8(b). An interchange mode $^{29}$ at $3.5 \mathrm{kHz}$ exists between $r=-5 \mathrm{~cm}$ and $r=0 \mathrm{~cm}$, both in the HEXTIP data and in the reconstructed camera data. The radial extension of the interchange mode structures is the same. The interchange mode in the case of the reconstructed emissivity is located at approximately $2 \mathrm{~cm}$ to the right-hand side of its radial position in the HEXTIP PSD profile. This displacement is less than $3.5 \mathrm{~cm}$ (spatial resolution of HEXTIP). Therefore, it could be due to spatial uncertainty in the HEXTIP measurements.

To visualize the mode structures and to estimate the size of structures, conditional average sampling (CAS) is performed by taking as trigger the signal of the reference plasma pixel of the reconstructed emissivity fluctuations at $z$ $=2 \mathrm{~cm}, r=0 \mathrm{~cm}$. A similar CAS technique is applied to the ion saturation current fluctuation data by taking as trigger the signal of a probe located at the center of the poloidal crosssection $(z=0 \mathrm{~cm}, r=0 \mathrm{~cm})$. This probe lies in the same flux tube going through the reference plasma pixel at $z$ $=2 \mathrm{~cm}, r=0 \mathrm{~cm}$. Figures 9 and 10 show nine consecutive frames of the CASed HEXTIP data and the reconstructed emissivity profile. In Fig. 9 the interchange mode structures are shown. The vertical and radial sizes of the mode structures are, respectively, $4 \pm 1.75$ and $6 \pm 1.75 \mathrm{~cm}$, taken as 

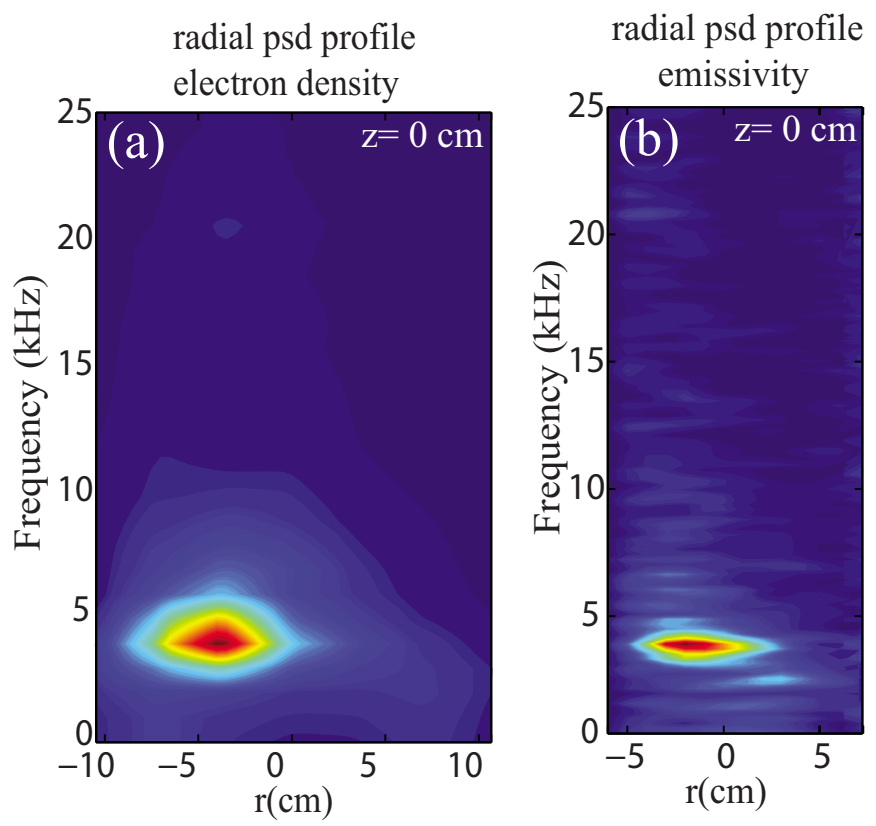

FIG. 8. (Color online) (a) Radial profile of the PSD of the ion saturation current fluctuations (HEXTIP). (b) Radial profile of the PSD of the reconstructed emissivity fluctuations.

the $F W H M$ of positive cells. Here, the uncertainty on the FWHM is taken as half of the tip spacing, which is $3.5 \mathrm{~cm}$ in the case of HEXTIP. The average distance of two positive peaks corresponding to the mode wavelength is $20 \pm 3.5 \mathrm{~cm}$. In Fig. 10, the spatial resolution of the CASed reconstructed emissivity is $2 \mathrm{~cm}$. Therefore, size measurements can be significantly more precise than with HEXTIP. The vertical and radial sizes of the positive peaks are $4 \pm 1$ and $5 \pm 1 \mathrm{~cm}$ and the wavelength is about $18 \pm 2 \mathrm{~cm}$. There is quite reasonable agreement between the CASed reconstructed emissivity and the CASed HEXTIP data in terms of the wavelength of the mode and sizes of structures. The tomographic reconstruction of TORPEX plasmas shows considerable similarities be-
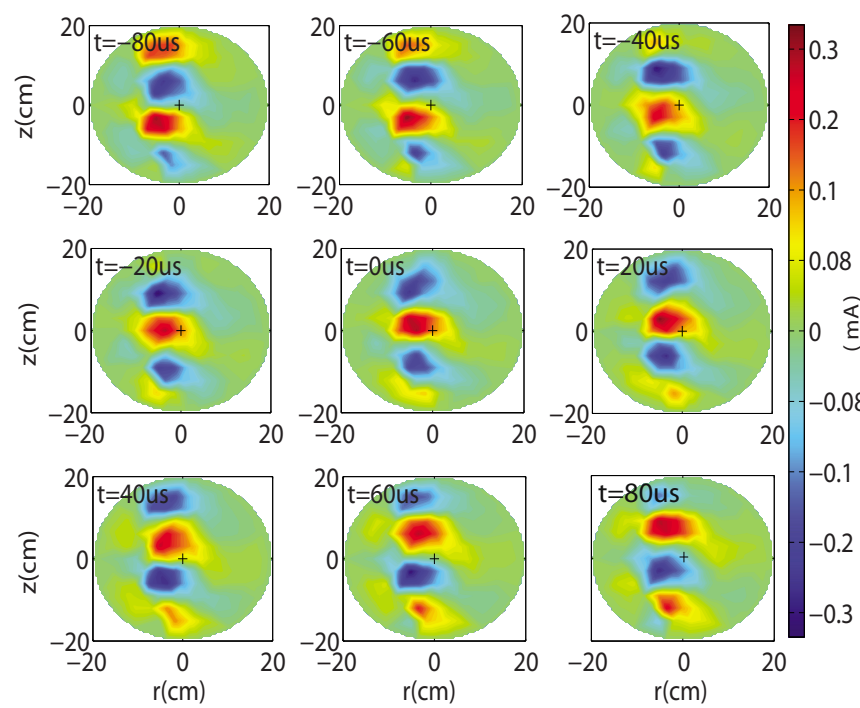

FIG. 9. (Color online) Nine consecutive frames of the CASed HEXTIP data by taking as trigger the signal of a probe located at the center of poloidal cross-section $(z=0 \mathrm{~cm}, r=0 \mathrm{~cm})$.

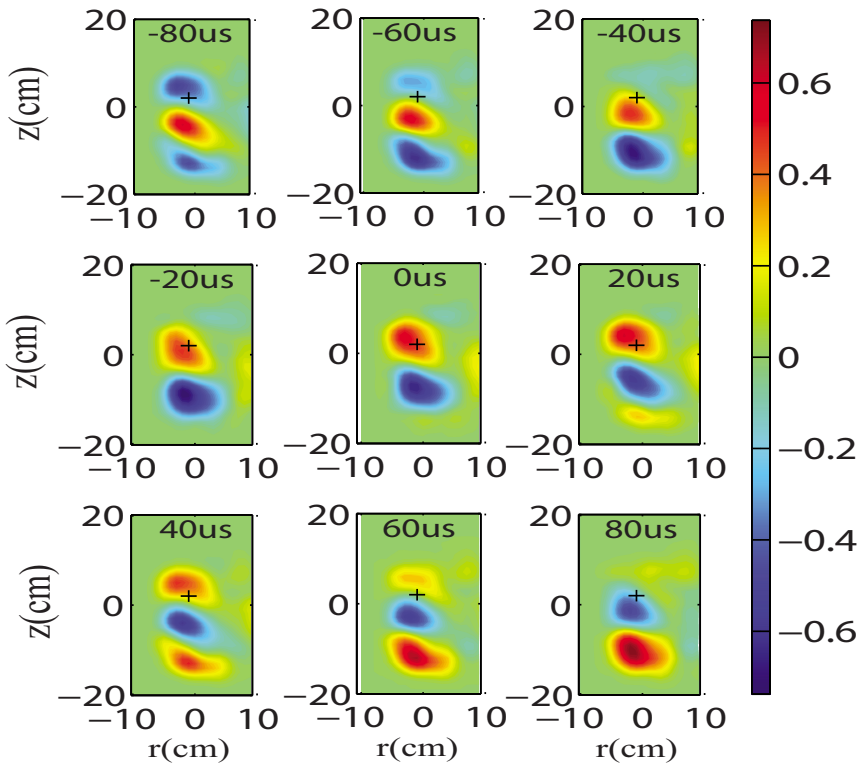

FIG. 10. (Color online) Nine consecutive frames of the CASed reconstructed emissivity profile by taking as trigger the signal of a plasma pixel of the reconstructed emissivity fluctuations at $z=2 \mathrm{~cm}, r=0 \mathrm{~cm}$.

tween ion saturation current and plasma emissivity in both time-averaged and fluctuation profiles. In addition, the camera can image small scale turbulent structures that are beyond the HEXTIP spatial resolution and cannot be studied with HEXTIP, but according to theory may have an important role in the turbulence dynamics. ${ }^{1}$ Consequently, the behavior of small scale turbulent structures can be investigated in plasma experiments using fast imaging as well as tomographic reconstruction.

To complete the comparison between HEXTIP data and the reconstructed emissivity movie, $k$-space Fourier analysis of each frame is performed. In principle, HEXTIP is able to detect structures with $k \leq 90\left(\mathrm{~m}^{-1}\right)$, while in the reconstructed emissivity profile smaller structures with $k \leq 300\left(\mathrm{~m}^{-1}\right)$ can be distinguished. Figures $11(\mathrm{a})$ and $11(\mathrm{~b})$ show, respectively, the $40 \mu$ s time-averaged $k$-space Fourier spectra of the fluctuation part of HEXTIP and the reconstructed emissivity profiles. In the $k$-space spectrum of the reconstructed emissivity, the $k$ associated with the inter-
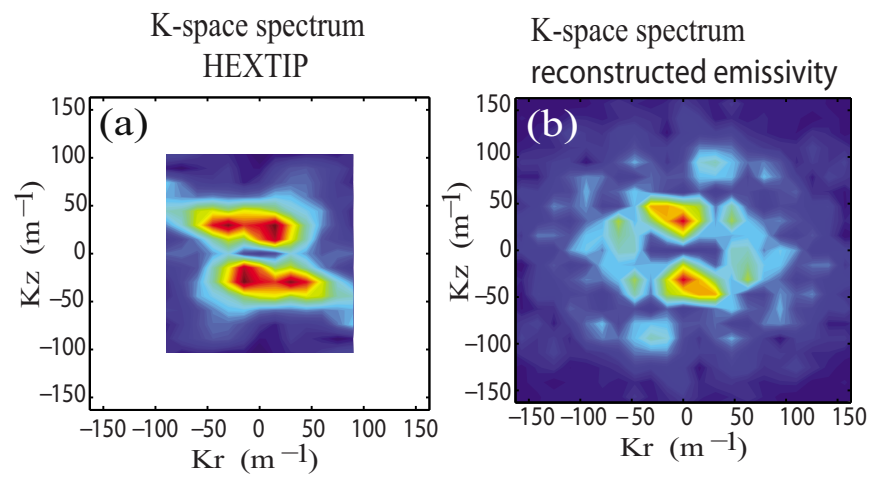

FIG. 11. (Color online) (a) $k$-space spectrum profile of HEXTIP data averaged over $40 \mu \mathrm{s}$. (b) $k$-space spectrum profile of reconstructed emissivity profile averaged over $40 \mu \mathrm{s}$. 
change mode is detected as well as smaller structures, well beyond the HEXTIP resolution limit.

\section{CONCLUSIONS}

To reconstruct the time-resolved poloidal emissivity of TORPEX plasma emission, a pixel method is considered and an optimized reconstruction procedure is applied to tangentially viewed camera images. The reconstruction is formalized by an overdetermined set of equations. The SVD approach is applied to perform the least squares method and to calculate the time-averaged emissivity profile of TORPEX plasmas. The tomographic reconstruction is accurate (i.e., $\mathrm{NR}<0.2$ ) up to $10 \%$ of the random noise and $30 \%$ of reflection and noise. The spatial resolution in the reconstructed emissivity profile is limited only by the plasma pixel size $(1 \mathrm{~cm})$ to $2 \mathrm{~cm}$ due to Nyquist theorem.

The plasma emissivity profiles and ion saturation current profiles are similar in terms of shape and position in interchange dominated plasmas. We show that in a large portion of the poloidal cross-section, the collisional radiative model provides a good approximation for the observed plasma emission. The emissivity fluctuations profile is deduced from the reconstructed emissivity by subtracting the timeaveraged reconstructed profile. The Fourier analysis of the time traces of the reconstructed emissivity shows coherent fluctuations at $3.5 \mathrm{kHz}$, which are associated with an interchange mode. This is in agreement with Fourier analysis of the ion saturation current fluctuations.

CAS is performed to visualize the mode structures and to estimate their sizes. There is reasonable agreement between the CASed reconstructed emissivity and the CASed HEXTIP data in terms of the mode wavelength and sizes of structures.

The tomographic reconstruction makes it possible to retrieve information about the temporal evolution of plasma emissivity, which is faded due to the line integration in the camera data. Therefore, the fast imaging of the plasma as a nonperturbative and high spatiotemporal resolution diagnostics can visualize small scale turbulent plasma structures in TORPEX. The theory predicts that small scale structures exist and may play an important role in the turbulence dynamics and in the associated transport. $k$-space Fourier analysis is applied to both HEXTIP data and reconstructed emissivity movie. The small scale turbulent plasma structures can be detected in $k$-space Fourier analysis of the reconstructed emissivity movie well beyond the spatial resolution of HEXTIP. The capability of visualization of small scale structures can provide the necessary experimental data to compare with the theory.

\section{ACKNOWLEDGMENTS}

We wish to acknowledge the helpful discussions with all TORPEX group members. We also thank Dr. Sergei Shibaev for providing the fast camera control and data transfer software. This work is partly supported by the Fonds National Suisse de la Recherche Scientifique.
${ }^{1}$ E. J. Doyle, W. A. Houlberg, Y. Kamada, V. Mukhovatov, T. H. Osborne, A. Polevoi, G. Bateman, J. W. Connor, J. G. Cordey, T. Fujita, X. Garbet, T. S. Hahm, L. D. Horton, A. E. Hubbard, F. Imbeaux, F. Jenko, J. E. Kinsey, Y. Kishimoto, J. Li, T. C. Luce, Y. Martin, M. Ossipenko, V. Parai, A. Peeters, T. L. Rhodes, J. E. Rice, C. M. Roach, V. Rozhansky, F. Ryter, G. Saibene, R. Sartori, A. C. C. Sips, J. A. Snipes, M. Sugihara, E. J. Synakowski, H. Takenaga, T. Takizuka, K. Thomsen, M. R. Wade, and H. R. Wilson, Nucl. Fusion 47, S18 (2007).

${ }^{2}$ S. J. Zweben, R. J. Maqueda, L. Terry, T. Munsat, J. R. Myra, D. D'Ippolito, D. A. Russell, J. A. Krommes, B. LeBlanc, T. Stoltzfus-Dueck, D. P. Stotler, K. M. Williams, C. E. Bush, R. Maingi, O. Grulke, S. A. Sabbagh, and A. E. White, Phys. Plasmas 13, 056114 (2006).

${ }^{3}$ G. Y. Antar, S. I. Krasheninnikov, P. Devynck, R. P. Doerner, E. M. Hollmann, J. A. Boedo, S. C. Luckhardt, and R. W. Conn, Phys. Rev. Lett. 87, 065001 (2001).

${ }^{4}$ A. Kirk, H. R. Wilson, G. F. Counsell, R. Akers, E. Arends, S. C. Cowley, J. Dowling, B. Lloyd, M. Price, and M. Walsh, Phys. Rev. Lett. 92, 245002 (2004)

${ }^{5}$ G. Y. Antar, J. H. Yu, and G. Tynan, Phys. Plasmas 14, 022301 (2007).

${ }^{6}$ J. A. Boedo, D. Rudakov, R. Moyer, S. Krasheninnikov, D. Whyte, G. McKee, G. Tynan, M. Schaffer, P. Stangeby, P. West, S. Allen, T. Evans, R. Fonck, E. Hollmann, A. Leonard, A. Mahdavi, G. Porter, M. Tillack, and G. Antar, Phys. Plasmas 8, 4826 (2001).

${ }^{7}$ S. J. Zweben, R. J. Maqueda, D. P. Stotler, A. Keesee, J. Boedo, C. E. Bush, S. M. Kaye, B. LeBlanc, J. L. Lowrance, V. J. Mastrocola, R. Maingi, N. Nishino, G. Renda, D. W. Swain, and J. B. Wilgen, Nucl. Fusion 44, 134 (2004).

${ }^{8}$ S. J. Zweben, D. P. Stotler, J. L. Terry, B. LaBombard, M. Greenwald, M. Muterspaugh, C. S. Pitcher, K. Hallatschek, R. J. Maqueda, B. Rogers, J. L. Lowrance, V. J. Mastrocola, and G. F. Renda, Phys. Plasmas 9, 1981 (2002).

${ }^{9}$ R. J. Maqueda, G. A. Wurden, S. J. Zweben, L. Roquemore, H. Kugel, D. Johnson, S. Kaye, S. Sabbagh, and R. Maingi, Rev. Sci. Instrum. 72, 931 (2001).

${ }^{10}$ A. Fasoli, B. Labit, M. McGrath, S. Müller, G. Plyushchev, M. Podesta, and F. M. Poli, Phys. Plasmas 13, 055902 (2006).

${ }^{11}$ I. Furno, B. Labit, M. Podesta, A. Fasoli, S. Müller, F. M. Poli, P. Ricci, C. Theiler, S. Brunner, A. Diallo, and J. Graves, Phys. Rev. Lett. 100, 055004 (2008).

${ }^{12}$ B. Labit, I. Furno, A. Fasoli, A. Diallo, S. Müller, G. Plyushchev, M. Podesta, and F. M. Poli, Phys. Rev. Lett. 98, 255002 (2007).

${ }^{13}$ S. Müller, A. Diallo, A. Fasoli, I. Furno, B. Labit, and M. Podesta, Phys. Plasmas 14, 110704 (2007)

${ }^{14}$ M. Podestà, A. Fasoli, B. Labit, M. McGrath, S. Müller, and F. M. Poli, Plasma Phys. Controlled Fusion 47, 1989 (2005).

${ }^{15}$ N. Nagayama, J. Appl. Phys. 62, 2702 (1987).

${ }^{16}$ J. F. Camacho and R. S. Granetz, Rev. Sci. Instrum. 57, 417 (1986).

${ }^{17}$ E. T. Powell, R. Kaita, and R. J. Fonck, Rev. Sci. Instrum. 61, 3301 (1990).

${ }^{18}$ T. Asai, T. Takahashi, T. Kiguchi, Y. Matsuzawa, and Y. Nogi, Phys. Plasmas 13, 072508 (2006).

${ }^{19}$ S. Banerjee and P. Vasu, Nucl. Fusion 49, 075032 (2009).

${ }^{20}$ A. K. Chattopadhyay, A. Anand, and C. V. S. Rao, Rev. Sci. Instrum. 76, 063502 (2005).

${ }^{21}$ N. Iwama, H. Yoshida, H. Takimoto, Y. Shen, S. Takamura, and T. Tsukishima, Appl. Phys. Lett. 54, 502 (1989).

${ }^{22}$ I. Furno, C. Angioni, F. Porcellia, H. Weisen, R. Behn, T. P. Goodman, M. A. Henderson, Z. A. Pietrzyk, A. Pochelon, H. Reimerdes, and E. Rossib, Nucl. Fusion 41, 403 (2001).

${ }^{23}$ M. Anton, H. Weisen, M. J. Dutch, W. von der Linden, F. Buhlmann, R. Chavan, B. Marletaz, P. Marmillod, and P. Paris, Plasma Phys. Controlled Fusion 38, 1849 (1996).

${ }^{24}$ D. Iraji, A. Diallo, A. Fasoli, I. Furno, and S. Shibaev, Rev. Sci. Instrum. 79, 10F508 (2008).

${ }^{25} \mathrm{See} \quad \mathrm{http} / / / \mathrm{sales}$.hamamatsu.com/en/products/electron-tube-division/ detectors/gated-image-intensifiers/part-c10880-03x.php.

${ }^{26}$ G. Chiodini, C. Riccardi, and M. Fontanesi, Rev. Sci. Instrum. 70, 2681 (1999).

${ }^{27}$ C. Theiler, A. Diallo, A. Fasoli, I. Furno, B. Labit, M. Podestà, and F. M. Poli, Phys. Plasmas 15, 042303 (2008).

${ }^{28}$ M. Goto, K. Sawada, and T. Fujimoto, Phys. Plasmas 9, 4316 (2002).

${ }^{29}$ F. M. Poli, P. Ricci, A. Fasoli, and M. Podesta, Phys. Plasmas 15, 032104 (2008). 